\title{
The petpace collar in action: detecting heart failure and response to treatment
}

\section{Patient condition and history}

Lucas, a 10year-old neutered male Beagle, suffers from Chronic Valvular Heart Disease (CVHD). He is treated with Benazepril and has been wearing the PetPace smart collar long-term to monitor his condition. Lucas' condition recently deteriorated over the course of several weeks, and his owner, Michelle Saltzman of Bedford, MA, took him to see his veterinarian, Dr. Patricia Berchtold, at Chelmsford Animal Hospital. Lucas was diagnosed to be in Congestive Heart Failure. He was treated first with a diuretic (furosemide), and then another drug (Vetmedin $®)$ was added. Following treatment Lucas showed significant clinical improvement.

\section{Monitoring data}

In the weeks preceding the onset of Lucas' Congestive Heart Failure, the PetPace collar detected gradual changes in several parameters, indicating the impending condition. To start, both Lucas' minimum pulse rate and minimum respiratory rate showed gradual, incremental increases over several weeks, as seen in the charts below Figure 1

\section{Case study I (Figure 2)}

Heart Rate Variability (HRV), an established indicator of various heart diseases, also showed a clear decrease during the same period of time (low HRV is indicative of poor cardiac health) Figure 3.
Volume 2 Issue 6 - 2015

\author{
Asaf Dagan \\ Chief Veterinary Scientist, PetPace LTD, USA
}

Correspondence: Asaf Dagan, Chief Veterinary Scientist, PetPace LTD, 25 Burlington Mall Road, Burlington, MA 01803 , USA, Tel: 054949 6955, 78। 325 8567,

Email asaf.dagan@petpace.com

Received: November 23, 2015 | Published: December 07, 2015

Treatment with furosemide, a diuretic drug, brought quick but short-lived improvement, as seen in the following HRV graph. The addition of a second drug, Vetmedin ${ }^{\circledR}$ (Pimobendan), produced a gradual but sustained improvement. These clinical changes were accurately reflected in the PetPace data.

\section{Case sstudy 2 (Figure 4)}

Activity data did not reveal statistically significant changes before the onset of Congestive Heart Failure, but a modest increase in activity was evident following medical treatment (Figure 5).

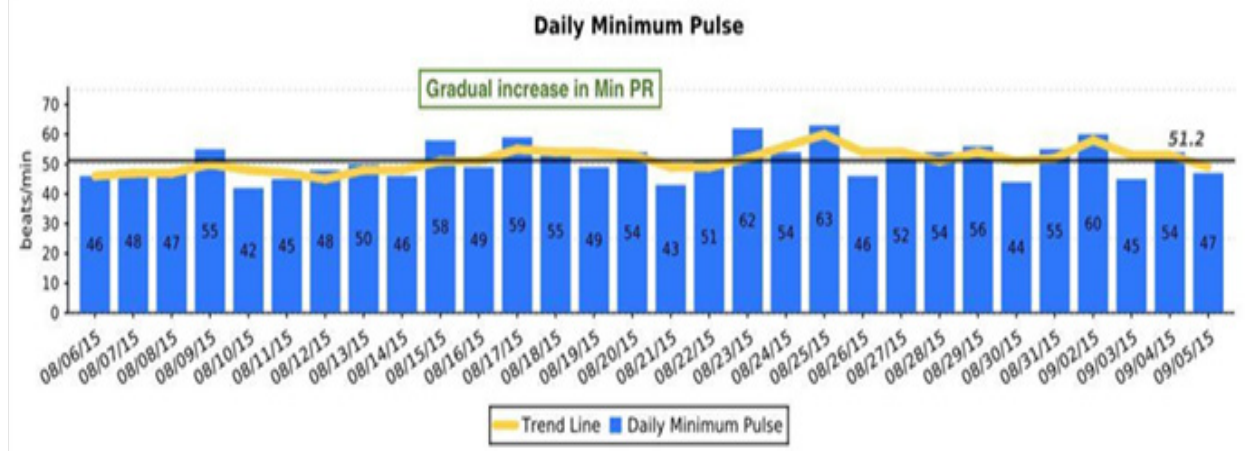

Figure I *Minimum pulse rate gradually increasing (yellow trend line) over I month prior to heart failure.

Daily Minimum Respiration

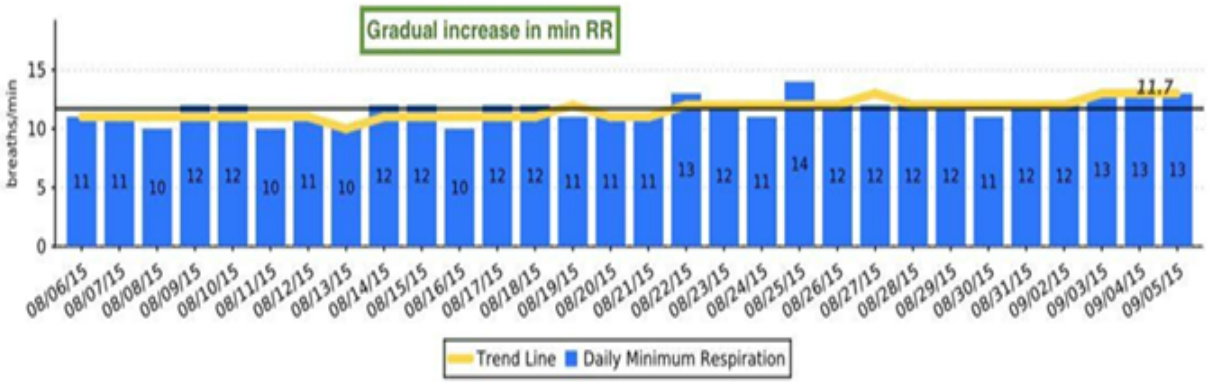

Figure 2 *Minimum respiratory rate gradually increasing (yellow trend line) over I month prior to heart failure. 
TRAINGULAR INDEX

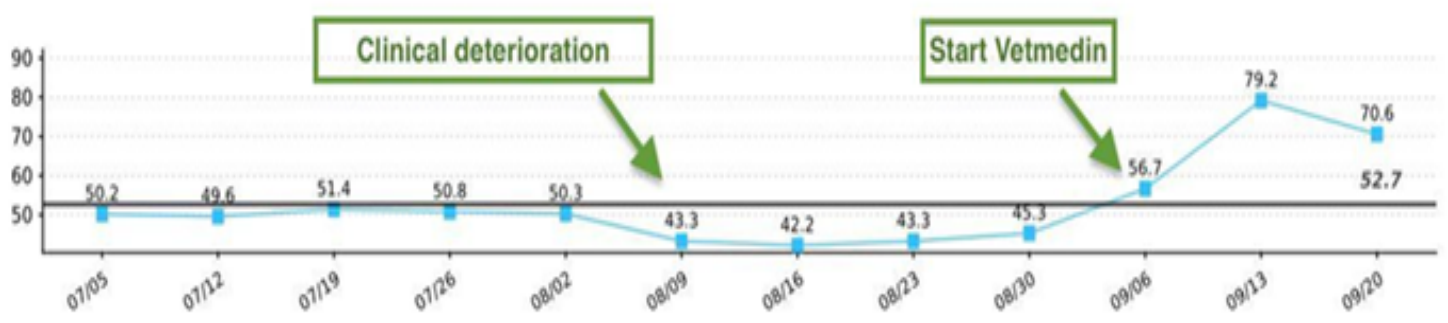

Figure 3 *Triangular Index, an example of HRV, showing approximately 15\% decrease in the weeks preceding congestive heart failure in a dog.

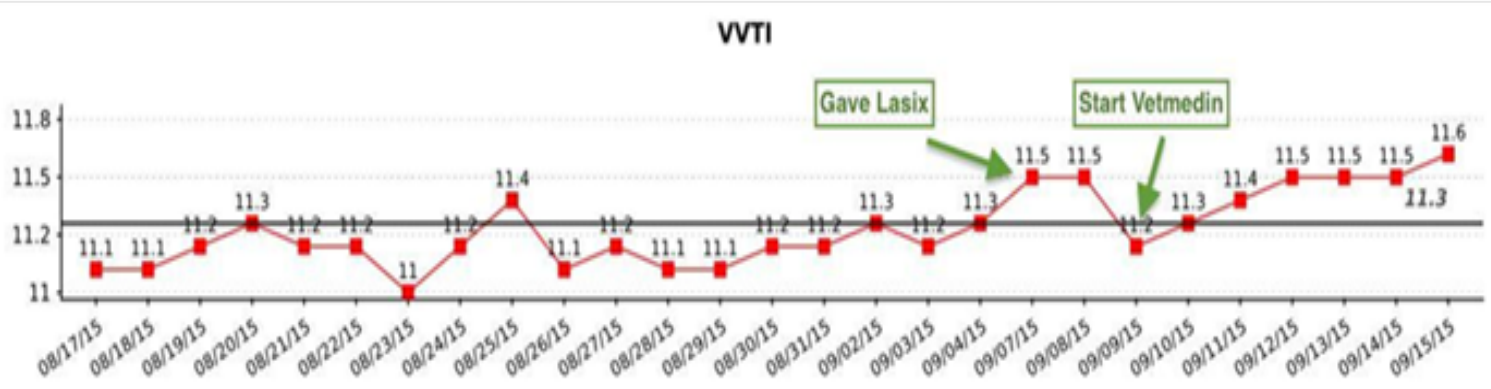

Figure 4 *VVTI, an example of $\mathrm{HRV}$ index, showing quick response to furosemide and a more gradual but sustained response to Vetmedin.

\section{Activity Trends}

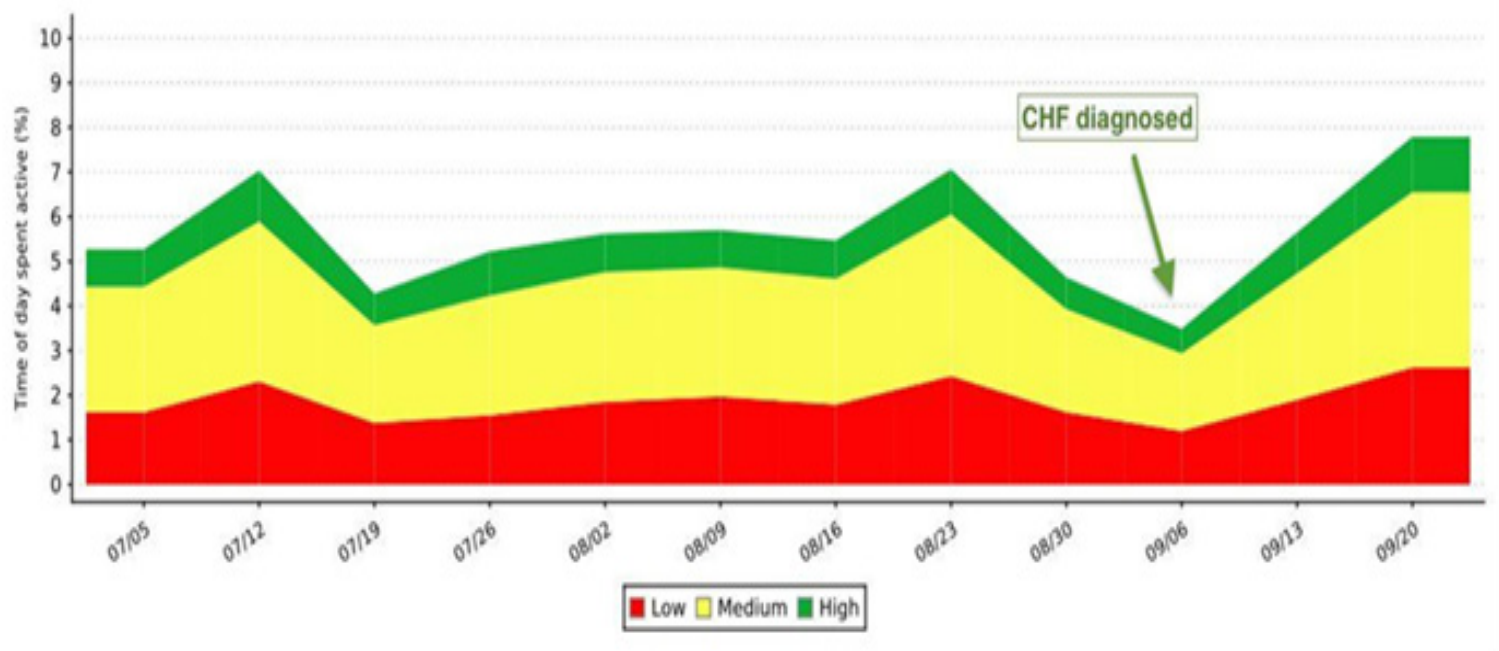

Figure 5 *Weekly activity chart from the weeks before and after heart failure diagnosis.

\section{Discussion}

Chronic Valvular Heart Disease (CVHD) is a common heart disease of older small-breed dogs, although it can appear in large breeds as well. It involves degenerative, non-inflammatory structural changes to the valves of the heart, which causes valve thickening and deformation. Some dogs with CVHD go on to develop Congestive Heart Failure, as was the case for Lucas. The gradual, incremental clinical deterioration in Lucas' condition was evident in trends of pulse and respiratory rates as well as HRV indices. On the other hand, changes in activity level during the same time period were not as distinct or statistically significant, underscoring the limited medical value of monitoring activity alone. Treatment with furosemide and Vetmedin ${ }^{\circledR}$ (Pimobendan) are considered standard of care for Congestive Heart Failure secondary to CVHD. The effect of both drugs can be clearly identified in the vital signs monitored by PetPace.

\section{Conclusion}

\section{Case study}

The PetPace collar is unique in its ability assist in the remote medical management of pets with heart disease. It provides early detection of trends preceding clinical deterioration, long before the pet shows overt symptoms. Moreover, it allows for close monitoring 
of the effect of certain drugs, potentially facilitating better clinical management.

Dr. Asaf Dagan, DVM, Diplomate ABVP (Canine and Feline practice), and PetPace's Chief Veterinarian, noted, "Clinicians can now use innovative wearable technology to remotely assess the condition of cardiac patients, detect worrisome trends and closely follow the effect of their chosen course of treatment. The impact of such capabilities on longevity and quality of life for patients with CVHD, and other conditions as well, may be tremendous".

Dr. Dan Ohad, DVM, PhD, Diplomate ACVIM and ECVIM (Cardiology) from Koret School of Veterinary Medicine, of the Hebrew University of Jerusalem, Israel, added, "The trends of data changes provided by the PetPace collar during hemodynamic deterioration and in response to pharmacotherapy carry an immense potential for both a timely diagnosis, far before the patient experiences life-threatening complications, and for timely therapeutic decision-making. This can enable prompt rectification of developments before they 'snowball' and potentially get out of control."

\section{Acknowledgements}

None.

\section{Conflict of interest}

Author declares that there is no conflict of interest. 